\title{
Limit periodic sets in polynomial Liénard equations
}

\author{
Stijn Luca, Freddy Dumortier
}

\author{
stijn.luca@uhasselt.be, freddy.dumortier@uhasselt.be \\ Hasselt University, Campus Diepenbeek, Agoralaan-Gebouw D, B-3590 Diepenbeek, Belgium
}

\begin{abstract}
In this paper we classify all limit periodic sets, as well bounded as unbounded ones, that occur in Liénard equations $\dot{x}=y, \dot{y}=P(x)+y Q(x)$, with $P$ and $Q$ polynomials of respective degrees $m$ and $n$ with $m n \geq 1$. We also classify the interior and exterior boundaries of the period annuli of such systems with special attention to the exterior boundary of the Hopf centers. We investigate under which conditions on $(m, n)$ certain configurations can occur.
\end{abstract}

Key words: Liénard equation, limit periodic set, period annulus, singularities.

2000 Mathematics Subject Classification: 34C07, 34C05, 34C37, 34D10.

\section{Introduction}

Consider a polynomial Liénard system of type $(m, n) ;(m, n) \in \mathbb{N} \times \mathbb{N}$ arbitrarily but fixed:

$$
X:\left\{\begin{array}{l}
\dot{x}=y \\
\dot{y}=P(x)+y Q(x),
\end{array}\right.
$$

where $P$ and $Q$ are polynomials of respective precise degrees $m$ and $n$. Shortly we refer to these systems as Liénard systems (of a certain type $(m, n)$ ). Using linear dilatations in $x, y$ and $t$, we can assume that $P$ and $Q$ are given by

$$
P(x)=-\left(A x^{m}+\sum_{i=0}^{m-1} a_{i} x^{i}\right), \quad Q(x)=-\left(x^{n}+\sum_{i=0}^{n-1} b_{i} x^{i}\right),
$$

with $A=1$ or -1 , if $m \neq 2 n+1$, and $A \in \mathbb{R} \backslash\{0\}$, if $m=2 n+1$.For a given degree $N$, Hilbert's 16th problem asks for a maximum number of limit cycles that an Nth degree polynomial vector field in the plane can have. Using the localisation method of Roussarie [7], the proof of the existence of such a finite upperbound can be reduced to the finite cyclicity of any limit periodic set occuring in the flow of a polynomial vector field of degree $N$. An attempt to solve the existence part of Hilbert's 16th problem for polynomial Liénard systems of a given type $(m, n)$ could hence start by listing all limit periodic sets occuring in Liénard systems of type $(m, n)$.

In this paper we want to describe all limit periodic sets that can occur in a polynomial Liénard system of type $(m, n)$, both bounded as unbounded. 
Preprint submitted at Qualitative Theory of Dynamical Systems. The final publication is available at http://link.springer.com/article/10.1007\\%2Fs12346-008-0019-9

We also give necessary conditions on $(m, n)$ for a limit periodic set to occur in $\bar{X} \in L^{(m, n)}(D)$. Further, we describe all possible boundaries of any period annulus occuring in a Liénard system, the bounded ones as well as the ones that extend to infinity.

First, in order to be able to use the localisation method of Roussarie and to study the behaviour of a polynomial Liénard equation at infinity, we need to compactify the phase plane and the chosen space of Liénard systems. We refer the reader to [2] and [3]. Let us describe shortly these compactifications.

By a Poincaré-Lyapunov compactification, the phase plane can be compactified in the so-called Poincaré-Lyapunov disc. For a general introduction to as well the Poincaré compactification as the Poincaré-Lyapunov compactification, we refer to [3] and [4]. Using an appropriate quasi-homogeneous compactification, the singularities at infinity will be of a rather simple nature. We use a Poincaré-Lyapunov compactification of type $(\alpha, \beta) \in \mathbb{N}_{1} \times \mathbb{N}_{1}$ to compactify the phase space in the Poincaré-Lyapunov disc of degree $(\alpha, \beta)$. This means that we set:

$$
x=\frac{\cos \theta}{v^{\alpha}}, \quad y=\frac{\sin \theta}{v^{\beta}},
$$

with $\theta \in \mathbb{S}^{1}$, and multiply the obtained vector field by a factor $v^{d}$ to extend the obtained vector field to the Poincaré-Lyapunov disc. Depending on $(m, n)$ the degree $(\alpha, \beta)$ and the power $d$ in the factor $v^{d}$ is chosen to be:

1. $(\alpha, \beta)=(1, n+1), d=n$, when $m \leq 2 n+1$,

2. $(\alpha, \beta)=(2, m+1), d=m-1$, when $m>2 n+1$ and $m$ is even,

3. $(\alpha, \beta)=\left(1, \frac{1}{2}(m+1)\right), d=(m-1) / 2$, when $m>2 n+1$ and $m$ is odd.

The compactification, indicated in the previous list, is called the appropriate compactification for the respective Liénard system of type $(m, n)$ [2]. The associated disc is called the appropriate Poincaré-Lyapunov disc and is denoted by $D^{(m, n)}$, or shortly $D$. We endow $D$ with the usual topology of a disc. The vector field on $D^{(m, n)}$ obtained from $X$, after such an appropriate Poincaré-Lyapunov compactification and multiplication by a factor $v^{d}$, is denoted as $\bar{X}$. The study near infinity of $\bar{X}$ can be done by means of different charts.

The space of all vector fields $\bar{X}$ on $D^{(m, n)}$ obtained, after an appropriate Poincaré-Lyapunov compactification, from a Liénard system $X$ of type $(m, n)$ with $P$ and $Q$ as in (2) will be denoted as $L^{(m, n)}(D)$. The systems obtained from a system (1) with $P$ or $Q$ zero constitute the boundary of this space of Liénard systems. These system are of Hamiltonian or singular type [2]. In using the localisation method for solving the existential part of Hilbert's 16th problem their limit periodic sets also have to be considered. In this paper we only pay attention to the limit periodic sets occuring in systems belonging to $L^{(m, n)}(D)$.

We remark that all results that are valid for systems:

$$
\left\{\begin{array}{l}
\dot{x}=y, \\
\dot{y}=P(x)+y Q(x)
\end{array}\right.
$$

can be transferred to similar results on:

$$
\left\{\begin{array}{l}
\dot{x}=Y-F(x), \\
\dot{Y}=P(x)
\end{array}\right.
$$


Preprint submitted at Qualitative Theory of Dynamical Systems. The final publication is available at http://link.springer.com/article/10.1007\\%2Fs12346-008-0019-9

where $F(x)=-\int_{0}^{x} Q(s) d s$. The transfer goes through the coordinate change:

$$
(y, x)=(Y-F(x), x),
$$

so that statements on $\{y \geq 0\}$ (resp. $\{y \leq 0\}$ ) will now hold on $\{y \geq F(x)\}$ (resp. $\{y \leq F(x)\}$ ). The transfer clearly works on the finite plane, but in fact the results including infinity still hold, since (see [2]) the coordinate change (3) can be extended to an analytic diffeomorphism on the appropriate PoincaréLyapunov disc.

\section{Singularities in Liénard systems}

Let $\bar{X} \in L^{(m, n)}(D)$, as in the introduction obtained after an appropriate Poincaré-Lyapunov compactification from a Liénard system $X$ of type $(m, n)$ (1), where $P$ and $Q$ are given like in (2).

In order to be able to describe all possible limit periodic sets, we first have to study all possible singularities of $\bar{X}$. The study of the singularities at infinity is already done in [3]. For sake of completeness and for later use, we recall the results. Figures 1, 2, 3 and 4 show the behaviour near infinity of $\bar{X}$ in the three cases: $m<2 n+1, m=2 n+1$ and $m>2 n+1$.

Note that the behaviour near infinity only depends on the degree $(m, n)$ and the coefficient $A$. In these pictures simple arrows on different curves near a singularity indicate that the singularity is hyperbolic, while for semi-hyperbolic singularities we use simple arrows for the centre behaviour and double arrows for the hyperbolic behaviour. Other singularities do not occur at infinity in the appropriate Poincaré-Lyapunov compactification.
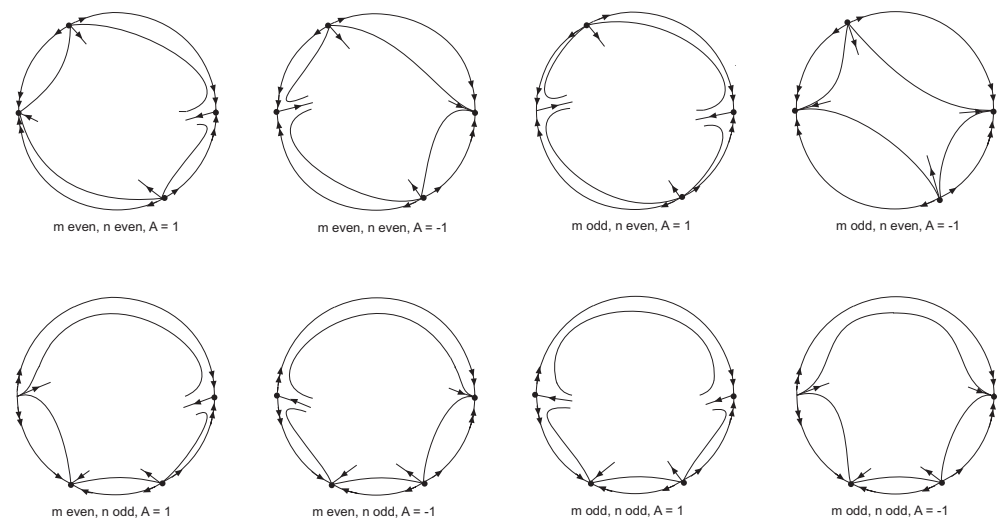

Figure 1: Behaviour near infinity for $m<2 n+1$ on the Poincaré-Lyapunov disc of degree $(1, n+1)$.

The study of the singularities of $\bar{X}$ situated in the interior of $D^{(m, n)}$ is equivalent to the study of the singularities of the original Lienard system $X$ of type $(m, n)$. Because $\dot{x}=y$ we say that the flow of $X$ points to the right above the $x$-axis and to the left below the $x$-axis. Regular orbits of $X$ will intersect the $x$-axis transversally. 
Preprint submitted at Qualitative Theory of Dynamical Systems. The final publication is available at http://link.springer.com/article/10.1007\\%2Fs12346-008-0019-9
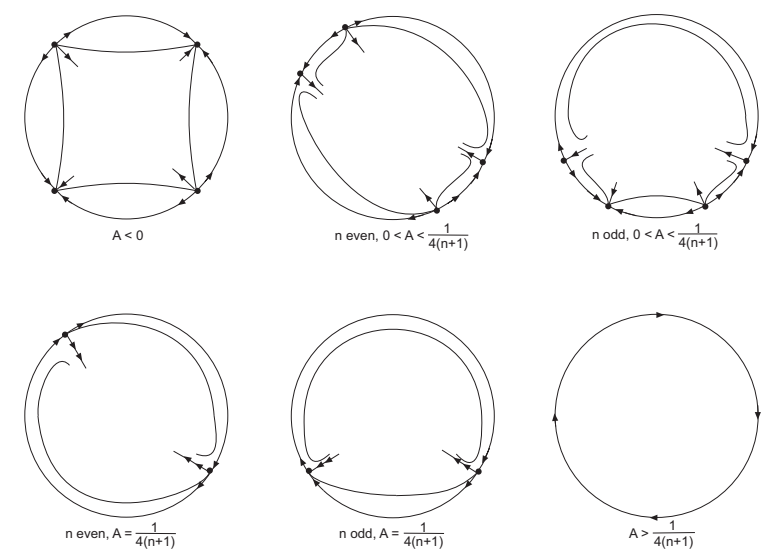

Figure 2: Behaviour near infinity for $m=2 n+1$ on the Poincaré-Lyapunov disc of degree $(1, n+1)$.
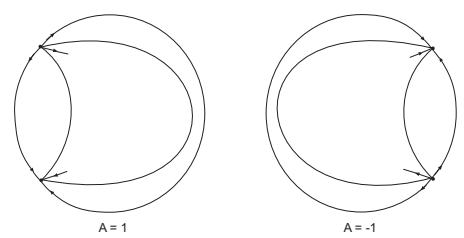

Figure 3: Behaviour near infinity for $m>2 n+1, m$ even, on the PoincaréLyapunov disc of degree $(2, m+1)$.
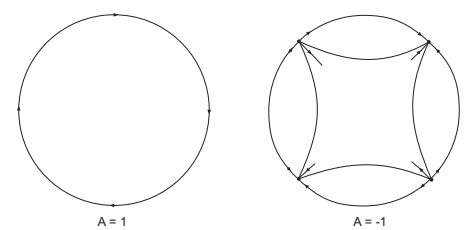

Figure 4: Behaviour near infinity for $m>2 n+1, m$ odd, on the PoincaréLyapunov disc of degree $\left(1, \frac{1}{2}(m+1)\right)$.

A singularity $s$ of $X$ is given by a point $s=\left(x_{0}, 0\right)$ with $P\left(x_{0}\right)=0$. The linear part of $X$ at $s$ is equal to

$$
\mathcal{A}:=\left(\begin{array}{cc}
0 & 1 \\
P^{\prime}\left(x_{0}\right) & Q\left(x_{0}\right)
\end{array}\right),
$$

with eigenvalues $\lambda_{1,2}=\frac{1}{2}\left(Q\left(x_{0}\right) \pm \sqrt{Q\left(x_{0}\right)^{2}+4 P^{\prime}\left(x_{0}\right)}\right)$ and corresponding eigenspaces $V_{1}$ and $V_{2}$ spanned by respectively $\left(1, \lambda_{1}\right)$ and $\left(1, \lambda_{2}\right)$. Moreover 
Preprint submitted at Qualitative Theory of Dynamical Systems. The final publication is available at http://link.springer.com/article/10.1007\\%2Fs12346-008-0019-9

$\lambda_{1} \lambda_{2}=-P^{\prime}\left(x_{0}\right)$. Further we write:

$$
\begin{aligned}
& P(x)=a\left(x-x_{0}\right)^{k}+o\left(x-x_{0}\right)^{k}, \\
& Q(x)=b\left(x-x_{0}\right)^{l}+o\left(x-x_{0}\right)^{l},
\end{aligned}
$$

where $a b \neq 0$ and with $(k, l) \in \mathbb{N}^{2}, 1 \leq k \leq m$ and $0 \leq l \leq n$. We call $(k, l)$ the Liénard degree of the singularity $s$.

The propositions below describe the singularities of a Liénard system (1) with respect to their Liénard degree and the coefficients $(a, b)$. We will present all possible phase portraits of the singularities of a Liénard system. A singulariy $s$ always lies on the $x$-axis. In case $s$ is not a focus nor a center, a dotted line indicates the direction in which orbits can approach the singularity or move away from it. Notice that in presenting the phase portraits, it is not possible to stress out the exact position of the orbits, nor their exact contact with the directions in which they approach $s$.

Proposition 1 If $k=1$ and:

1. if $a>0, s$ is a hyperbolic saddle as in Figure 5 (a).

2. if $a<0, l=0$ and $b^{2}+4 a \geq 0$, then $s$ is a stable node when $b<0$ and an unstable node when $b>0$. When $b^{2}+4 a>0$, all orbits except two will approach the singularity along the eigenspace that corresponds with the eigenvalue that is smallest in absolute value (see Figure 5, (b) and (c)). In case $b^{2}+4 a=0$ all orbits will approach $s$ along the unique eigenspace (see Figure 5, (d) and $5($ e)). In particular s cannot be a star node.

3. if $a<0, l=0$ and $b^{2}+4 a<0$, then $s$ is a stable focus when $b<0$ and an unstable focus when $b>0$ (see Figure 5, $(f)$ and $(g)$ ).

4. if $a<0$ and $l>0$, then $s$ is linearly a center. The singularity is a focus or a center (see Figure $5(h))$.

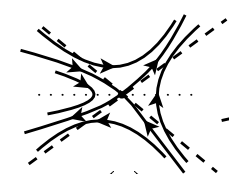

(a)

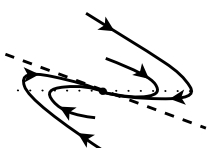

(e)

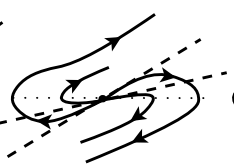

(b)

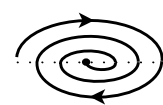

(f)

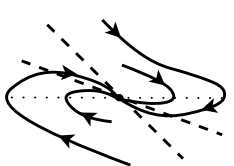

(c)

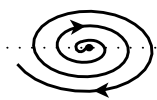

(g)

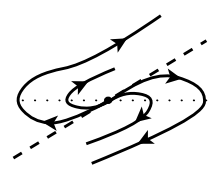

(d)

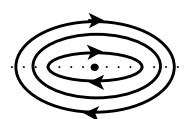

(h)

Figure 5: Phase portraits of non-degenerate singularities.

Proof: If $s$ is not a center, the type of the singularity can be deduced from the linear part $\mathcal{A}$. In case $\mathcal{A}$ has a center in $s$, it can be a center or focus for $X$. We are then left with the well-known center-focus problem. The directions in which the orbits approach the singularity can be found by blowing up, see for instance [6]. Phase portraits are obtained by using the fact that the flow of $X$ points to the right above the $x$-axis and to the left below the $x$-axis. 
Preprint submitted at Qualitative Theory of Dynamical Systems. The final publication is available at http://link.springer.com/article/10.1007\\%2Fs12346-008-0019-9

Proposition 2 The singularity $s$ of $X$ is semi-hyperbolic if and only if $k \geq 2$ and $l=0$. Every center manifold has at $s$ a contact of order $k-1$ with the $x$-axis and on it, the vector field behaves like

$$
\dot{x}=-\frac{a}{b}\left(x-x_{0}\right)^{k}+o\left(x-x_{0}\right)^{k} .
$$

Depending on whether $b$ is positive or negative there is an unstable respectively stable manifold tangent to $y=b\left(x-x_{0}\right)$. Denoting $Q(x)-b=c\left(x-x_{0}\right)^{p}+$ $o\left(x-x_{0}\right)^{p}$ for some $p \geq 1$, the (un)stable manifold has a contact at $s$ of order $\min \{k-1, p\}$ with $y=b\left(x-x_{0}\right)$. Furthermore:

1. if $k$ is odd and $a>0$ the singularity is of saddle-type (Figure 6, (a) and (b)).

2. if $k$ is odd and $a<0$ the singularity is a stable node when $b>0$ and an unstable node when $b<0$ (Figure 6 , (c) and (d)).

3. if $k$ is even the singularity is a saddle-node (Figure 6, (e), (f), (g) and (h)).

Moreover orbits not lying on the (un)stable manifold and adherent to s belong to a center manifold, having at $s$ a contact of order $k-1$ with the $x$-axis.

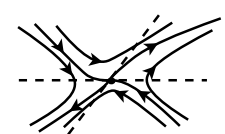

(a)

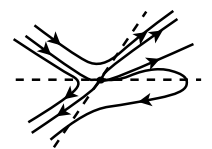

(e)

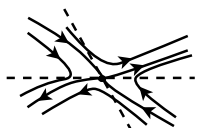

(b)

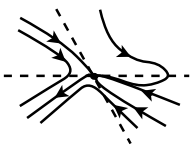

(f)

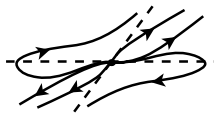

(c)

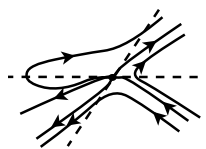

(g)

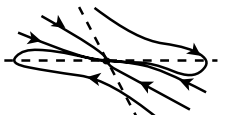

(d)

Figure 6: Phase portraits of semi-hyperbolic singularities.

Proof: The singularity is semi-hyperbolic if and only if $\lambda_{1} \lambda_{2}=0, \lambda_{1}+\lambda_{2} \neq 0$ which is equivalent to $P^{\prime}\left(x_{0}\right)=0, Q\left(x_{0}\right)=b \neq 0$. The linear part of $X$ at $s$ is given by:

$$
\mathcal{A}=\left(\begin{array}{ll}
0 & 1 \\
0 & b
\end{array}\right)
$$

with eigenvalues $0, b$ and respective eigenspaces $y=0$ and $y=b\left(x-x_{0}\right)$.

The center manifold theorem ensures the existence of at least one $C^{\infty}$ manifold $y=c(x)$ tangent to the $x$-axis and invariant under the flow of $X$ (see e.g. $[4])$, i.e.

$$
c^{\prime}(x) c(x)=P(x)+c(x) Q(x) \quad \text { or } \quad c(x)\left(-b+O\left(x-x_{0}\right)\right)=P(x)
$$

such that $P(x)=O\left(x-x_{0}\right)^{k}$ implies $c(x)=O\left(x-x_{0}\right)^{k}$. Comparing coefficients one sees that

$$
c(x)=-\frac{a}{b}\left(x-x_{0}\right)^{k}+o\left(x-x_{0}\right)^{k} .
$$


Preprint submitted at Qualitative Theory of Dynamical Systems. The final publication is available at http://link.springer.com/article/10.1007\\%2Fs12346-008-0019-9

Suppose now $y=s(x)$ is the (unique) invariant manifold tangent to the (un)stable direction $y=b\left(x-x_{0}\right)$. Just as before the invariance implies

$$
s(x)\left(s^{\prime}(x)-Q(x)\right)=P(x) .
$$

Substituting $Q(x)=b+O\left(x-x_{0}\right)^{p}$ and $s(x)=b\left(x-x_{0}\right)+O\left(x-x_{0}\right)^{2}$ one obtains

$$
\left(b+O\left(x-x_{0}\right)\right)\left(s^{\prime}(x)-b\right)=O\left(x-x_{0}\right)^{k-1}+O\left(x-x_{0}\right)^{p},
$$

such that $s^{\prime}(x)-b=O\left(x-x_{0}\right)^{\min \{k-1, p\}}$, meaning that at $x_{0}$ the contact between $y=b\left(x-x_{0}\right)$ and $y=s(x)$ is of order $\min \{k-1, p\}$.

If $\gamma$ is an orbit not lying on the unique (un)stable manifold and it approaches $s$, then we know that it lies on a center manifold [4]. Because all center manifolds are mutually infinitely tangent [4], $\gamma$ has to have at $s$ a contact of order $k-1$ with the $x$-axis.

The phase portraits are easily obtained using the fact that $\dot{x}=y$.

Proposition 3 The singularity $s$ of $X$ is nilpotent if and only if $k \geq 2$ and $l \geq 1$. Moreover:

1. if $k=2 p$ is even and $k<2 l+1$, the singularity is a cusp for which the separatrices have at $s$ a contact of order $p$ (Figure 7, (a) and (b)).

2. if $k$ is even and $k>2 l+1$, the singularity is a saddle-node. Orbits can only approach $s$ along the $x$-axis having at $s$ mutual contact of order $l$ (Figure 7, (c), (d), (e) and (f)).

3. if $k=2 p+1$ is odd and $a>0$, then the singularity is a saddle where the separatrices are tangent to the $x$-axis having at $s$ mutual contact of order $p$ when $k \leq 2 l+1$ and of order $l$ when $k>2 l+1$ (Figure $7(g))$.

4. if $k$ is odd and $a<0$ and:

(a) if $k<2 l+1$, or $k=2 l+1$ and $b^{2}+4 a(l+1)<0$, then the singularity is a focus or center (Figure 7, ( $h),(i)$ and $(j))$,

(b) if $l$ is odd and either $k>2 l+1$, or $k=2 l+1$ and $b^{2}+4 a(l+1) \geq 0$, then $s$ is a singularity with one elliptic and one hyperbolic sector where orbits can only approach $s$ along the $x$-axis having at s mutual contact of order l (Figure 7, (k) and (l) ),

(c) if $l$ is even and either $k>2 l+1$, or $k=2 l+1$ and $b^{2}+4 a(l+1) \geq 0$ then $s$ is an attractive node when $b<0$ and a repelling node when $b>0$. All orbits will approach $s$ along the $x$-axis having at $s$ mutual contact of order l (Figure 7, (m) and (n)).

Proof: If $Q\left(x_{0}\right)=0$ and $P^{\prime}\left(x_{0}\right)=0$, we see immediately that the linear part of $X$ is nilpotent.

The study of the singularity relies on quasi-homogeneous blow-up. In case $k \leq 2 l+1$ and $k$ is odd, one uses the blow up

$$
x=u, \quad y=u^{q} \bar{y},
$$




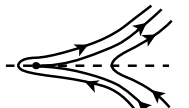

(a)

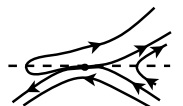

(e)

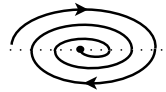

(i)

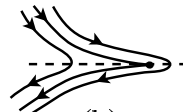

(b)

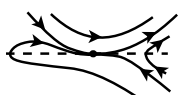

(f)

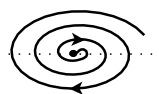

(j)

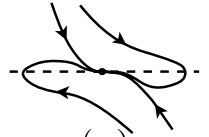

$(\mathrm{m})$

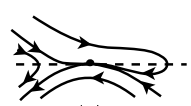

(c)

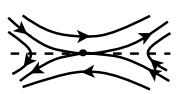

(g)

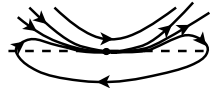

$(\mathrm{k})$

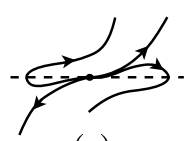

(n)

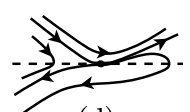

(d)

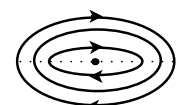

(h)

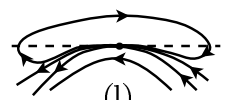

(1)

Figure 7: Phase portraits of nilpotent singularities.

with $q=\frac{k+1}{2}$ and we divide by $u^{q-1}$. If $k$ is even, one uses:

$$
x=u^{2}, \quad y=u^{k+1} \bar{y},
$$

and we divide by $u^{k-1}$. In case $k>2 l+1$, one uses the blow up:

$$
x=u, \quad y=u^{l+1} \bar{y}
$$

and we divide by $u^{l}$.

In the directional charts $\{\bar{y}= \pm 1\}$, one won't find any singularity in the origin. We can therefore restrict our study to the $\{u= \pm 1\}$-charts. However using the transformations (7) and (8), the information found in the $\{u=1\}-$ chart also covers the information in the $\{u=-1\}$-chart. We refer to [4] where all calculations are present.

Let us clarify the statements about the contacts. In blow-up coordinates the $\bar{y}$-axis is invariant under the flow and corresponds to the origin in the original coordinates. Suppose $y=y(x)$ is an orbit that approaches the singularity $s$, then this orbit will correspond to an orbit $\bar{y}=\bar{y}(u)$ not lying on the $\bar{y}$-axis that approaches a singularity on the $\bar{y}$-axis.

If $k \leq 2 l+1$, one finds no singularities at the origin after blow up [4]. Therefore $\bar{y}(u)=\alpha+o(1), u \rightarrow 0$ with $\alpha \neq 0$. After blowing down, we get $y(x)=\alpha x^{\frac{k+1}{2}}+o\left(x^{\frac{k+1}{2}}\right), x \rightarrow 0$.

In case $k>2 l+1$ one finds, after blowing up, two singularities on the $\bar{y}-$ axis [4]. The origin is a semi-hyperbolic singularity with a unique (un)stable manifold lying on the $\bar{y}$-axis. All other orbits are given by $\bar{y}(u)=o\left(u^{k-2 l-2}\right)$, $u \rightarrow 0$. Blowing down gives $y(x)=o\left(x^{k-l-1}\right), x \rightarrow 0$. The other singularity is a saddle having a unique invariant manifold, transverse to the $\bar{y}$-axis, given by $\bar{y}(u)=\alpha+o(1), u \rightarrow 0$ with $\alpha \neq 0$. Blowing down yields $y(x)=o\left(x^{l}\right), x \rightarrow 0$. Because $k>2 l+1$, we get certainly $y(x)=o\left(x^{l}\right), x \rightarrow 0$ in both cases.

The previous propositions classify the singularities of Liénard systems (1) according to their Liénard degree $(k, l)$ and the coefficients $(a, b)$, defined in 
Preprint submitted at Qualitative Theory of Dynamical Systems. The final publication is available at http://link.springer.com/article/10.1007\\%2Fs12346-008-0019-9

(5). It is clear that a singularity of Liénard degree $(k, l)$ can only occur in a Liénard system of type $(m, n)$ when $m \geq k$ and $n \geq l$. As a direct consequence of the former propositions, we can state the following theorem that presents all possible singularities of a Liénard system of type $(m, n) \in \mathbb{N}^{2}$.

Theorem 4 A Liénard system $X$ of type $(m, n)$ as defined in (1) has at most $m$ singularities, where we count a singularity $\left(x_{0}, 0\right), k+1$ times when $P\left(x_{0}\right)=$ $P^{\prime}\left(x_{0}\right)=\cdots=P^{(k)}\left(x_{0}\right)=0$. Denote by $s$ a singularity of $X$, then:

1. $s$ can be a hyperbolic singularity: a saddle, a node (no star node) or a focus, see Figure 5, (a)-(g). Near a node, all orbits approach s along one of the eigenspaces of the linear part of $X$ at $s$.

2. when $n \geq 1, s$ can be a linear center, i.e. a center or a focus, see Figure $5,(f),(g)$ or $(h)$.

3. if $m \geq 2, s$ can be a saddle-node. When $m \geq 3, s$ is possibly a semihyperbolic node or a semi-hyperbolic saddle. The unique (un)stable manifold intersects the $x$-axis transversally. The center manifolds will have at the singularity a contact of order at most $m$ with the $x$-axis. See Figure 6 .

4. if $m \geq 2, n \geq 1, s$ can be a cusp, when $m \geq 3, n \geq 1, s$ can be a nilpotent focus, center, saddle or a singularity with an elliptic sector, if $m \geq 4$, $n \geq 1, s$ can be a nilpotent saddle-node, when $m \geq 5, n \geq 2$, s can be a nilpotent node. In case $s$ is not a center nor a focus, orbits can only approach the singularity s along the $x$-axis. See Figure $\%$.

\section{$3 \quad$ Limit periodic sets in Liénard systems}

After a study of all possible singularities occuring in a Liénard system $\bar{X} \in$ $L^{(m, n)}(D)$, we are able to describe all possible limit periodic sets occuring in $\bar{X}$. We will also describe necessary conditions on $(m, n)$ for a certain limit periodic set to occur in $\bar{X}$.

For being able to treat limit periodic sets, we will endow $L^{(m, n)}(D)$ with a topology. We endow $L^{(m, n)}(D)$ with the coefficient topology. This is the topology $\mathcal{T}^{(m, n)}$ such that the following map is a homeomorphism:

$$
\begin{aligned}
\pi:\left(L^{(m, n)}(D), \mathcal{T}^{(m, n)}\right) & \mapsto(\mathcal{B}, \mathcal{U}) \\
\bar{X} & \mapsto\left(A, a_{m-1}, \ldots, a_{0}, b_{n-1}, \ldots, b_{0}\right),
\end{aligned}
$$

associating to each $\bar{X} \in L^{(m, n)}(D)$, the coefficients

$$
\left(A, a_{m-1}, \ldots, a_{0}, b_{n-1}, \ldots, b_{0}\right)
$$

of the polynomials $P$ and $Q$ of the original system $X(1)$ and where $\mathcal{B}$ is given by $\{-1,1\} \times \mathbb{R}^{n+m}$, when $m \neq 2 n+1$, and $\mathbb{R} \backslash\{0\} \times \mathbb{R}^{n+m}$, when $m=2 n+1$, equipped with the induced topology $\mathcal{U}$ from $\mathbb{R}^{m+n+1}$.

Define $\mathcal{H}(D)$ as the set of all non-empty compact subsets of $D$ provided with the Hausdorff metric, i.e.

$$
d_{H}\left(K_{1}, K_{2}\right)=\sup \left\{\sup _{x \in K_{1}} d\left(x, K_{2}\right), \sup _{y \in K_{2}} d\left(K_{1}, y\right)\right\} ; \quad K_{1}, K_{2} \in \mathcal{H}\left(\mathbb{R}^{2}\right),
$$


Preprint submitted at Qualitative Theory of Dynamical Systems. The final publication is available at http://link.springer.com/article/10.1007\\%2Fs12346-008-0019-9

where $d(a, K)=\inf _{x \in K}\{\rho(a, x)\}, \rho$ the Euclidean metric on $\mathbb{R}^{2}$.

A compact subset $\mathcal{L}$ of $D$ is said to be a limit periodic set of $\bar{X} \in L^{(m, n)}(D)$ if for each $\varepsilon>0$ and for each neighbourhood $V$ of $\bar{X}$, there exists an $\bar{Y} \in V$ whose flow contains a limit cycle $\gamma$ such that $d_{H}(\gamma, \mathcal{L})<\varepsilon$. Limit periodic sets were first introduced in [5] and further worked out in [8].

A limit periodic set can only be a singularity situated in $D \backslash \partial D$, a periodic orbit or a graphic of $\bar{X}[8]$. A graphic of $\bar{X}$ consists of a finite (non-zero) number of singularities $s_{1}, \ldots, s_{r}$, not necessarily different, and a finite (nonzero) number of regular orbits $\gamma_{1}, \ldots, \gamma_{r}$ connecting them in the sense that for $i=1, \ldots, r: \omega\left(\gamma_{i}\right)=s_{i+1}$ and $\alpha\left(\gamma_{i+1}\right)=s_{i}$, where $s_{r+1}=s_{1}$. We take care of taking $r$ as small as possible avoiding useless coincidences in the graphic. A graphic that is homeomorphic with the unit circle $S^{1}$ is called a cycle. Depending on whether a limit periodic set, a periodic orbit or a graphic fits in $D \backslash \partial D$ or not, we call it bounded resp. unbounded.

An oriented path in a graphic starting at $A$ and ending at $B$ constitutes a (singular) connection between two points $A$ and $B$ on $\mathcal{L}$. We speak of a connection from $A$ to $B$.

Let us define a passage $\left\{\mathcal{O}_{\omega}, s, \mathcal{O}_{\alpha}\right\}$ at a singularity $s$ of $X_{\lambda_{0}}$ as the union of $s$ together with a regular orbit $\mathcal{O}_{\omega}$ with $s$ as $\omega$-limit and a regular orbit $\mathcal{O}_{\alpha}$ with $s$ as $\alpha$-limit. It is clear that every singularity $s$ of a graphic has to possess at least one passage.

Let $\varphi: A \subset[-1,1] \mapsto D$ be a $C^{\omega}$ function on an open connected subset $A \subset$ $[-1,1]$. Here $[-1,1]$ is endowed with the induced topology from $\mathbb{R}$. Take $\Sigma=$ $\varphi(A)$ with the induced topology. If $\varphi: A \subset[-1,1] \mapsto \Sigma$ is a homeomorphism such that for every $a \in A, \varphi^{\prime}(a)$ and $\bar{X}(\varphi(a))$ are linearly independent, then $\Sigma$ is called a transverse section of $\bar{X}$.

From the fact that $\dot{x}=y$ in a Liénard system, it is easily seen that for abritrarily $x$, the sets $\Sigma_{x}^{+}=\{(x, y) \in D \mid y>0\}$ and $\Sigma_{x}^{-}=\{(x, y) \in D \mid y<0\}$ are transverse sections for any $\bar{X} \in L^{(m, n)}(D)$.

The following proposition is an easy consequence of the proof of the PoincaréBendixson theorem. The proof of it can be found in [8].

Proposition 5 Suppose $\mathcal{L}$ is a limit periodic set of $\bar{X}$ and $\Sigma$ is a transverse section of $\bar{X}$. Then $\Sigma$ intersects $\mathcal{L}$ in at most one point.

\subsection{Bounded limit periodic sets}

Let us describe the bounded limit periodic sets $\mathcal{L}$ occuring in a $\bar{X} \in L^{(m, n)}(D)$ for certain $(m, n) \in \mathbb{N}^{2}$.

If $\mathcal{L}$ is a bounded periodic orbit, one easily sees that it will be $C^{\omega}$ diffeomorphic to a circle cutting the $x$-axis transversally at two distinct points.

We already know that all singularities of a bounded graphic have to lie on the $x$-axis and have to possess at least one passage $\left\{\mathcal{O}_{\omega}, s, \mathcal{O}_{\alpha}\right\}$. Locally both $\mathcal{O}_{\alpha}$ and $\mathcal{O}_{\omega}$ have the option to lie under or above the $x$-axis. We come to four different passages:

i. a left-right-passage (lr-passage): when both $\mathcal{O}_{\alpha}$ and $\mathcal{O}_{\omega}$ lie above the $x$-axis,

ii. a right-left-passage (rl-passage): when both $\mathcal{O}_{\alpha}$ and $\mathcal{O}_{\omega}$ lie under the $x$-axis, 
Preprint submitted at Qualitative Theory of Dynamical Systems. The final publication is available at http://link.springer.com/article/10.1007 \\%2Fs12346-008-0019-9

iii. an upgoing-passage: when $\mathcal{O}_{\alpha}$ lies above and $\mathcal{O}_{\omega}$ under the $x$-axis,

iv. a downgoing-passage: when $\mathcal{O}_{\alpha}$ lies under and $\mathcal{O}_{\omega}$ above the $x$-axis.

We call lr-passages and rl-passages also horizontal passages and the upgoingand downgoing passages also vertical passages. In Figure 8 the four kind of passages are illustrated.

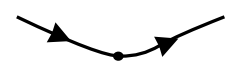

(a)

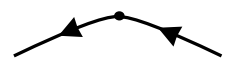

(b)

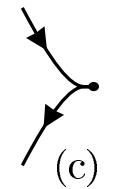

(c)

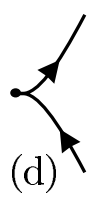

Figure 8: Horizontal and vertical passages of a singularity of a Lienard system.

Only the singularities containing passages can be part of a graphic. Let us give an overview, based on the results in Section 2, of all singularities possessing passages.

We begin with the singularities containing a horizontal passage. Saddles contain as well a lr-passage as a rl-passage (Figure 9). Saddle-nodes and singularities with one elliptic sector contain either a lr-passage (Figure 10) or a rl-passage (Figure 11).

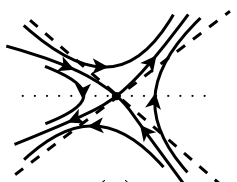

(a)

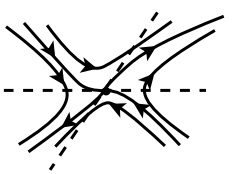

(b)

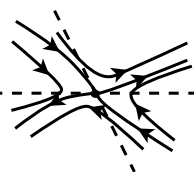

(c)

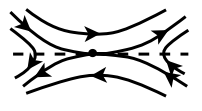

(d)

Figure 9: Saddles contain both lr- and rl-passages as well as both upgoing-and downgoing-passages.

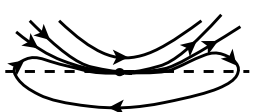

(a)

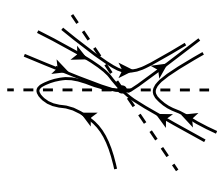

(b)

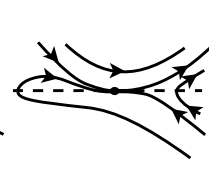

(c)

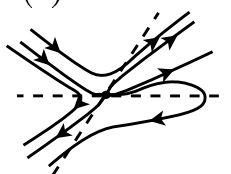

(d)

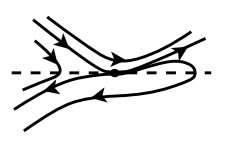

(e)

Figure 10: Singularities containing lr-passages but no rl-passages.

The singularities with a vertical passage are the saddles, containing upgoingas well as donwgoing-passages (Figure 9), the singularities in Figure 12 containing upgoing but no downgoing-passages and the singularities in Figure 13, containing downgoing- but no upgoing-passages.

Remark that the singularities in Figures 10 and 11, (b) and (c), also contain upgoing-passages while the ones in (d) and (e) also contain downgoing-passages. The singularities in Figures 12 and 13, (b) and (c), contain lr-passages while the ones in (d) and (e) contain rl-passages. Saddles contain all four kind of 
Preprint submitted at Qualitative Theory of Dynamical Systems. The final publication is available at http://link.springer.com/article/10.1007\\%2Fs12346-008-0019-9

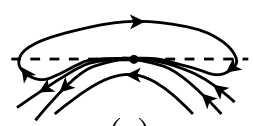

(a)

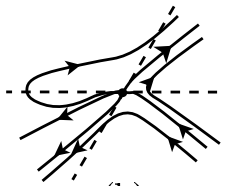

(b)

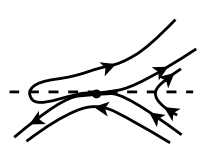

(c)

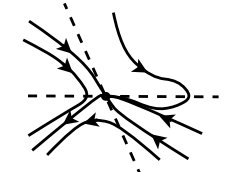

(d)

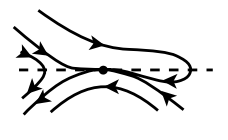

(e)

Figure 11: Singularities containing rl-passages but no lr-passages.

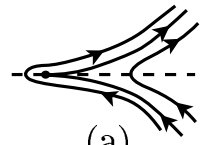

(a)

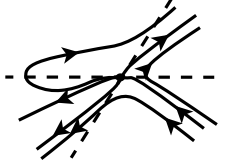

(b)

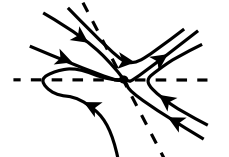

(c)

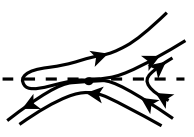

(d)

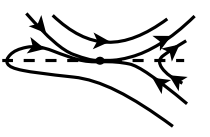

(e)

Figure 12: Singularities containing upgoing-passages.

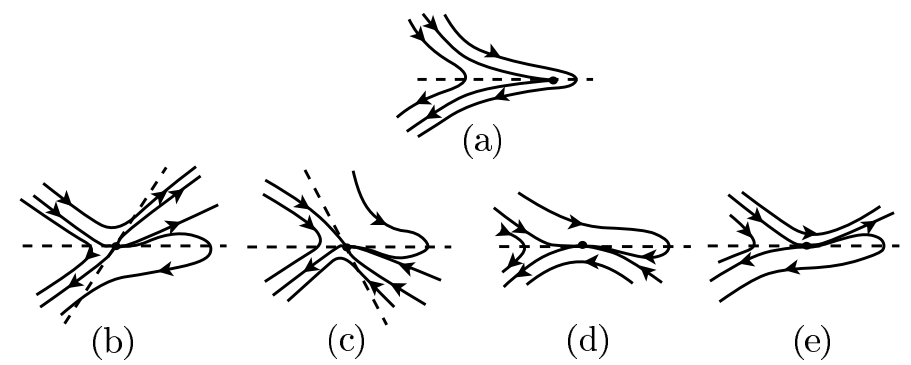

Figure 13: Singularities containing downgoing-passages.

passages. Furthermore horizontal passages nor vertical passages have to be unique. For instance saddle-nodes can contain infinitely many horizontal or vertical passages.

As a matter of example, we list all possible bounded limit periodic sets containing at most 2 singularities. Note that an exact position of the orbits or exact contacts cannot be illustrated in these pictures. To shorten the list of the graphics, we work modulo reflections with respect to the $x$-axis, $y$-axis or the origin, i.e. up to the transformations

$$
(y, t) \mapsto(-y,-t), \quad(x, t) \mapsto(-x,-t), \quad(x, y) \mapsto(-x,-y) .
$$

First of all a bounded limit periodic set $\mathcal{L}$ can be a bounded periodic orbit or 


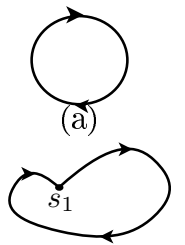

(d)

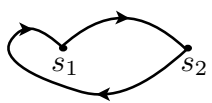

(g)

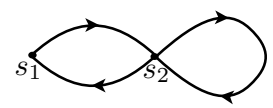

(j) $\dot{s_{1}}$

(b)

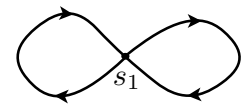

(e)

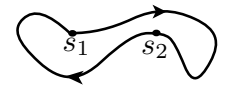

(h)

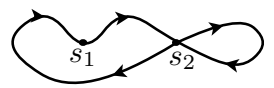

(k)

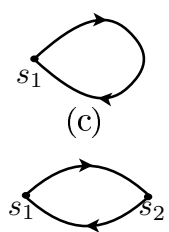

(f)

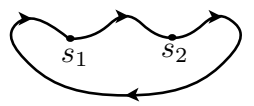

(i)

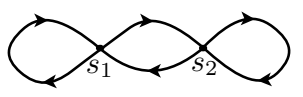

(1)

Figure 14: Possible bounded limit periodic sets containing at most two singularities.

a singularity, Figure 14, (a) and (b). Let $\mathcal{L}$ be a bounded graphic. If $\mathcal{L}$ contains one singularity, then depending on the kind of passage of the singularity, we find 3 different pictures illustrated in Figure 14, (c), (d) and (e). If $\mathcal{L}$ contains two singularities $\left\{s_{1}, s_{2}\right\}$, one distincts another 7 different pictures according to the kind of passages at $s_{1}$ and $s_{2}$ that lie on $\mathcal{L}$.

Concerning the possible topological types of the different singularities in Figure 14, we can say the following, making use of the above presentation of all singularities containing passages. In Figure 14 (b) every topological type can a priori occur. Of course if the singularity is a saddle, then it cannot be a hyperbolic one, but it can e.g. be a nilpotent saddle of codimension 3 and similar for a focus. In Figure 14 (e), $s_{1}$ has as well a lr-passage as a rl-passage implying that it has to be a saddle (hyperbolic or non-hyperbolic). Singularities with a vertical passage on $\mathcal{L}$ are possibly saddles, saddle-nodes or cusps. Those with a horizontal passage on $\mathcal{L}$ can only be saddles, saddle-nodes or singularities with one elliptic sector.The following theorem describes any bounded limit periodic set.

Theorem 6 Suppose $\mathcal{L}$ is a bounded limit periodic set of $\bar{X} \in L^{(m, n)}(D)$. Then $\mathcal{L}$ is a non-hyperbolic singularity of $\bar{X}$ situated in $D \backslash \partial D$ or it is the union of two continuous graphs $y=h_{1}(x)$ and $y=h_{2}(x), x \in\left[a_{0}, b_{0}\right], a_{0}<b_{0}$ such that:

i. $\forall x \in\left[a_{0}, b_{0}\right]: h_{1}(x) \geq 0$ and $h_{2}(x) \leq 0$. Moreover $h_{1}\left(a_{0}\right)=h_{2}\left(a_{0}\right)=0$ and $h_{1}\left(b_{0}\right)=h_{2}\left(b_{0}\right)=0$.

ii. for $x \in] a_{0}, b_{0}\left[, h_{1}(x) h_{2}(x)=0\right.$ if and only if $(x, 0)$ is a singularity of $\bar{X}$ lying on $\mathcal{L}$.

In this way, each graphic is an union of (singular) cycles and this decomposition is unique. Moreover concerning the singularities, we have the following:

a. the zeros of $h_{1}$ (resp. $h_{2}$ ) with abscis lying in $] a_{0}, b_{0}[$ can only be saddles or one of the singularities presented in Figure 10 (resp. Figure 11), 
Preprint submitted at Qualitative Theory of Dynamical Systems. The final publication is available at http://link.springer.com/article/10.1007\\%2Fs12346-008-0019-9

b. if $\left(a_{0}, 0\right)$ (resp. $\left.\left(b_{0}, 0\right)\right)$ is a singularity, then it has to be a saddle or one of the singularities presented in Figure 12 (resp. Figure 13),

c. if $\left.h_{1}(x)=h_{2}(x)=0, x \in\right] a_{0}, b_{0}[$, then $(x, 0)$ can only be a saddle (see Figure 9).

Proof: Denote the singularities and regular orbits that constitute $\mathcal{L}$ as $s_{1}, \ldots, s_{r}$ and $\gamma_{1}, \ldots, \gamma_{r}$, with $r$ minimal. We suppose that $\alpha\left(\gamma_{i}\right)=s_{i}$ and $\omega\left(\gamma_{i}\right)=s_{i+1}$. We accept that some $s_{i}=s_{j}$ for $i \neq j$, but let us show that it is justified to assume that $\gamma_{i} \neq \gamma_{j}$ for every $i \neq j \in\{1, \ldots, n\}$. If not, suppose $\gamma_{i}=\gamma_{j}$ for some $i \neq j$, then also $s_{i}=s_{j}$ and $s_{i+1}=s_{j+1}$. For $\mathcal{L}$ being closed there has to correspond (singular) connections $\Omega_{i}$ from $s_{i+1}$ to $s_{i}$ and $\Omega_{j}$ from $s_{j+1}$ to $s_{j}$. These connections have to coincide because otherwise they would result in two intersections of $\mathcal{L}$ with some transveral $\Sigma_{x}^{+}$or $\Sigma_{x}^{-}$contradicting Proposition 5 . So $\mathcal{L}_{i}=\left\{s_{i}, s_{i+1}, \gamma_{i}, \Omega_{i}\right\}$ describes the same graphic as $\mathcal{L}_{j}=\left\{s_{j}, s_{j+1}, \gamma_{j}, \Omega_{j}\right\}$ implying that the chosen configuration is not minimal, i.e. $r$ is not as small as possible.

Because $\mathcal{L}$ is compact, it is meaningful to set $a_{0}=\min \{x \mid(x, y) \in \mathcal{L}\}$ and $b_{0}=\max \{x \mid(x, y) \in \mathcal{L}\}$. In following the graphic $\mathcal{L}$ from $\left(a_{0}, 0\right)$ to $\left(a_{0}, 0\right)$, it can be shown that the $x$-component of the points on $\mathcal{L}$ first has to increase in a monotone way until reaching $b_{0}$ before it decreases again to $a_{0}$. If not, some $x$-values would have to be crossed at least twice in an upgoing or downgoing movement. This is not allowed because of Proposition 5 and the minimality requirements on $r$.

As such it is clear that the (singular) connection from $\left(a_{0}, 0\right)$ to $\left(b_{0}, 0\right)$ remains in $\{y \geq 0\}$ while the (singular) connection from $\left(b_{0}, 0\right)$ to $\left(a_{0}, 0\right)$ remains in $\{y \leq 0\}$. These connections being connected they have to cut each line $\{x\} \times \mathbb{R}$, for $x \in] a_{0}, b_{0}[$, and because of Proposition 5 and the minimality assumption on $r$, they both cut each $\{x\} \times \mathbb{R}$ exactly once, implying that they can be represented by graphs $\left\{y=h_{i}(x)\right\}$ of functions $h_{i}$ defined on $\left[a_{0}, b_{0}\right]$.

Clearly $h_{i}$ is continuous, even analytic, at points $\left.x \in\right] a_{0}, b_{0}\left[\right.$, where $h_{i}(x) \neq 0$. At points $\left.x_{0} \in\right] a_{0}, b_{0}$ [ where $h_{1}\left(x_{0}\right)=0$ or $h_{2}\left(x_{0}\right)=0$, the Liénard equation has to be singular. Using the classification of the singularities given in Propositions 1,2 and 3 , it is clear that $h_{1}$ and $h_{2}$ are also continuous at such points. Similar arguments can be used at $a_{0}$ and $b_{0}$.

The other statements of the theorem are also immediate consequences of Propositions 1, 2 and 3.

\subsection{Unbounded limit periodic sets}

Concerning the unbounded limit periodic sets, we have the following theorem.

Theorem 7 Suppose $\bar{X} \in L^{(m, n)}(D)$ has an unbounded limit periodic set $\mathcal{L}$. Then one of the below mentioned conditions has to be satisfied; A denotes the highest order coefficient of $P$ in (1).

1. When

(a) $m=2 n+1, A>\frac{1}{4(n+1)}$ or,

(b) $m>2 n+1, m$ odd and $A=1$,

$\mathcal{L}$ is given by a periodic orbit lying at infinity, Figure 15 (a). 
Preprint submitted at Qualitative Theory of Dynamical Systems. The final publication is available at http://link.springer.com/article/10.1007 \\%2Fs12346-008-0019-9

2. When

$$
m=2 n+1, n \text { even, } A=\frac{1}{4(n+1)},
$$

$\mathcal{L}$ looks like in Figure 15 (b). The two singularities are saddle-nodes with a center behaviour at infinity and a repelling behaviour on their hyperbolic separatrices.

3. When

$$
m=2 n+1, n \text { odd, } A=\frac{1}{4(n+1)}
$$

$\mathcal{L}$ looks like in Figure 15, (c), (d1) or (d2). The singularities $s_{1}$ and $s_{2}$ at infinity are both saddle-nodes with a center seperatix at infinity. The behaviour on the hyperbolic separatrix of $s_{1}$ is attractive, while that of $s_{2}$ is repelling.

4. When

$$
m=2 n+1, n \text { odd }, 0<A<\frac{1}{4(n+1)},
$$

$\mathcal{L}$ looks like in Figure 15, (d1) or (d2). In this case the singularities $s_{1}$ and $s_{2}$ are both hyperbolic saddles.

5. When

$$
m<2 n+1, m \text { odd, } n \text { odd and } A=1,
$$

$\mathcal{L}$ looks like in Figure 15, (e1) or (e2) containing at infinity two semihyperbolic saddles $s_{1}$ and $s_{2}$ such that $s_{1}$ (resp. $s_{2}$ ) has an unstable (resp. stable) separatrix lying at infinity and an attractive (resp. repelling) center separatrix in $D \backslash \partial D$.

Moreover in all cases the singularities on $\mathcal{L}$ that are situated inside $D \backslash \partial D$ have to be saddles or one of the singularities represented in Figure 11.

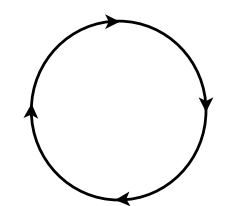

(a)

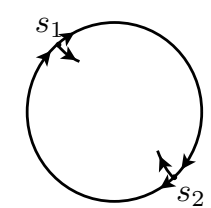

(b)

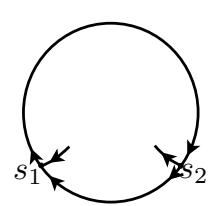

(c)

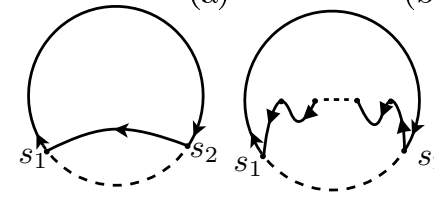

(d1)

$(\mathrm{d} 2)$

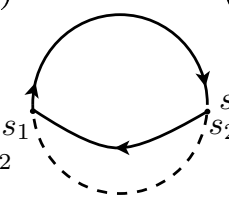

(e1)

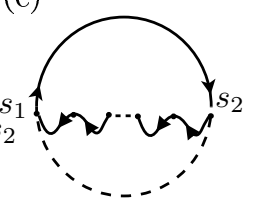

(e2)

Figure 15: All possible unbounded limit periodic sets (we do not pay attention to the exact position and contact of orbits nor to the exact nature of the singularities). 
Preprint submitted at Qualitative Theory of Dynamical Systems. The final publication is available at http://link.springer.com/article/10.1007\\%2Fs12346-008-0019-9

Proof: The study of the behaviour of $\bar{X}$ near infinity immediately implies that the above mentioned cases are the only possible ones for which $\bar{X}$ can have a limit periodic set. Indeed we can distinguish two cases: $\mathcal{L}$ includes the whole circle at infinity or only part of it. In the first case, it can be a regular periodic orbit at infinity or a graphic at infinity that only contains singularities possessing a passage at infinity. It is obvious that this is only possible when $m=2 n+1$, $A \geq \frac{1}{4(n+1)}$ or when $m>2 n+1$, m odd and $A=1$.

When a limit periodic set $\mathcal{L}$ is not lying enterily at infinity, it has to contain at least one connection on $\partial D$ bordered by two singularities $p_{1}$ and $p_{2}$ that both contain a passage. For $\mathcal{L}$ being closed, $p_{1}$ and $p_{2}$ have to possess a hyperbolic sector such that the flow on the separatrix lying in $D \backslash \partial D$ is attractive in one singularity and repelling in the other. This is only possible either when $m<$ $2 n+1, m$ odd, $n$ odd and $A=1$ or when $m=2 n+1, n$ odd, $0<A \leq \frac{1}{4(n+1)}$.

The topological type of the singularities at infinity immediately follows from the study of the behaviour near infinity of $\bar{X}$ in the above mentioned cases. In Figure $15,(\mathrm{~d} 1),(\mathrm{d} 2),(\mathrm{e} 1)$ and $(\mathrm{e} 2), \mathcal{L}$ contains the upper semicircle at infinity. Therefore for not being in contradiction with proposition 5 the remaining part of $\mathcal{L}$ has to be situated in the half plane $\{y \leq 0\}$. In particular all regular orbits of $\mathcal{L}$ lying in $D \backslash \partial D$ are situated below the $x$-axis implying that all singularities on $\mathcal{L}$ not lying at infinity have to contain a rl-passage.

\subsection{Occurence of limit periodic sets}

Let $\bar{X} \in L^{(m, n)}(D)$. We try to find necessary conditions on $(m, n)$ for a limit periodic set $\mathcal{L}$ to occur in the flow of $\bar{X}$.

Suppose first $\mathcal{L}$ is a bounded limit periodic set of $\bar{X}$. If $\mathcal{L}$ is a singularity with Liénard degree $(k, l)$, then obviously $m \geq k$ and $n \geq l$. When $\mathcal{L}$ is a bounded periodic orbit, then $m n \geq 1$. Indeed it is generally known that inside a periodic orbit, there has to exist a singularity together with a point where the divergence of $X$, i.e. $Q(x)$, disappears.

Suppose now that $\mathcal{L}$ is not a singularity nor a periodic orbit. Denote by $\left\{s_{1}, \ldots, s_{t}\right\}$ with $s_{i}=\left(p_{i}, 0\right)$ the singularities on $\mathcal{L}$. We know from Theorem 6 , that $\mathcal{L}$ is, in an unique way, the union of cycles that can only meet each other in a saddle. Denote by $n_{0}$ the number of cycles constituting $\mathcal{L}$. The Liénard degree $(K, L)$ of $\mathcal{L}$ is defined as:

$$
(K, L)=\left(\sum_{i=1}^{t} k_{i}, \sum_{i=1}^{t} l_{i}\right)
$$

where $\left(k_{i}, l_{i}\right)$ is the Liénard degree of the singularity $s_{i}$ on $\mathcal{L}$, counting each singularity of $\mathcal{L}$ once.

A singularity $s_{i}=\left(p_{i}, 0\right)$ induces a local behaviour on the vector field $X$ restricted to the $x$-axis. Because $X(x, 0)=(0, P(x))$ this behaviour is completely determined by the local behaviour of $P(x)$ at the zero $p_{i}$. In particular a singularity $s_{i}$ induces a sign on $P$ in a right- and left semi-neighbourhood of $p_{i}$. For instance, if $s_{i}$ is a saddle (see Figure 9 ), then $P$ is positive in a right semi-neighbourhood of $p_{i}$ and negative in a left semi-neighbourhood of $p_{i}$. Moreover if the endpoints $a_{0}$ and $b_{0}$ of $\mathcal{L}$ are not singularities, then it is clear that $P\left(a_{0}\right)>0$ and $P\left(b_{0}\right)<0$. From the intermediate value theorem, it 
Preprint submitted at Qualitative Theory of Dynamical Systems. The final publication is available at http://link.springer.com/article/10.1007\\%2Fs12346-008-0019-9

follows that there exists a minimum number $m_{0}$ of singularities that $X$ is forced to have for $P$ being continuous on $\left[a_{0}, b_{0}\right]$.

The following proposition now states necessary conditions for $\mathcal{L}$ to occur in the flow of $X$.

Theorem 8 Suppose $\mathcal{L}$ is a bounded limit periodic set of an $\bar{X} \in L^{(m, n)}(D)$, then:

1. if $\mathcal{L}$ is a singularity with Liénard degree $(k, l)$, then $m \geq k$ and $n \geq l$,

2. if $\mathcal{L}$ is a periodic orbit, then $m n \geq 1$,

3. if $\mathcal{L}$ is a graphic, if $m_{0}$ and $n_{0}$ are defined as above and $(K, L)$ is the Liénard degree of $\mathcal{L}$, then $m$ and $n$ have to satisfy

$$
m \geq K+m_{0} \quad \text { and } \quad n \geq L+n_{0} .
$$

Proof: Suppose $\mathcal{L}$ is a graphic. Then the degree of the polynomial $P$ has to be at least the number of its zeros, counted with their multiplicity. Thus $m \geq K+m_{0}$. Furthermore in each area enclosed by a cycle, there has to exist a point where the divergence of $X$, i.e. $Q(x)$, is zero such that $n \geq L+n_{0}$.

When $\bar{X}$ contains an unbounded limit periodic set, $m$ has to be odd and $A>0$ such that $\lim _{x \rightarrow-\infty} P(x)<0$ and $\lim _{x \rightarrow+\infty} P(x)>0$. Moreover if $\mathcal{L}$ contains a singularity $s=(p, 0)$ in $D \backslash \partial D$, then it induces a local behaviour on $P$ near $p$ as before. Again the continuity of $P$, forces $X$ to have a minimum number $m_{0}$ of singularities. Further we define the Liénard degree of an unbounded limit periodic set $\mathcal{L}$ as:

$$
(K, L)=\left(\sum_{i=1}^{t} k_{i}, \sum_{i=1}^{t} l_{i}\right),
$$

where $\left(k_{i}, l_{i}\right)_{i}, i=1, \ldots, t$ are the Liénard degrees of the (different) singularities on $\mathcal{L}$ situated in $D \backslash \partial D$. As a direct consequence of Theorem 7 , we can state necessary conditions on $(m, n)$ for an unbounded limit periodic set $\mathcal{L}$ to occur in a Liénard system of type $(m, n)$.

Theorem 9 Suppose $\mathcal{L}$ is an unbounded limit periodic set of $\bar{X} \in L^{(m, n)}(D)$. Then if $m_{0}$ is defined as above and $(K, L)$ is the Liénard degree of $\mathcal{L}$, the following applies:

1. if $\mathcal{L}$ is a periodic orbit at infinity, then $m \geq 2 n+1, m$ odd,

2. if $\mathcal{L}$ is like in Figure $15(b)$, then $m=2 n+1, n$ even,

3. if $\mathcal{L}$ is like in Figure 15, (c) or (d1), then $m=2 n+1$ and $n$ is odd,

4. if $\mathcal{L}$ is like in Figure 15 (d2), then $m=2 n+1, n$ is odd, $m \geq K+m_{0}$ and $n \geq L$,

5. if $\mathcal{L}$ is like in Figure $15(e 1)$, then $m<2 n+1, m$ and $n$ are odd,

6. if $\mathcal{L}$ is like in Figure 15 (e2), then $m<2 n+1, m$ and $n$ are odd, $m \geq$ $K+m_{0}$ and $n \geq L$. 
Preprint submitted at Qualitative Theory of Dynamical Systems. The final publication is available at http://link.springer.com/article/10.1007\\%2Fs12346-008-0019-9

One can now ask oneself whether a limit periodic set does occur in a system satisfying the minimal conditions given in Theorems 8 and 9. Let us verify this in some examples. A singularity with a Liénard degree $(k, l)$ can indeed occur in a Liénard system of type $(k, l)$ as already discussed in Section 2. Periodic orbits obviously occur in the Liénard system:

$$
X:\left\{\begin{array}{l}
\dot{x}=y, \\
\dot{y}=-x+x y
\end{array}\right.
$$

having, by symmetry reasons, a center at the origin. Furthermore, from Section 2 we know that the system:

$$
X:\left\{\begin{aligned}
\dot{x} & =y \\
\dot{y} & =-\frac{1}{2} x+y
\end{aligned}\right.
$$

has a strong stable focus in the origin and a periodic orbit lying at infinity. However a bounded periodic orbit isn't possible here because the divergence of $X$ does not disappear anywhere.

Concerning a loop as in Figure 14 (c) that is based at a hyperbolic saddle, we found that $m$ has to be at least 2 and $n$ at least 1 . It is well known that such a hyperbolic saddle-loop does indeed occur in a Liénard system of type $(2,1)$, see for instance [6].

We finish with an example containing an unbounded 2-saddle cycle, where the saddles at infinity are both hyperbolic. Consider the Liénard system

$$
\left\{\begin{array}{l}
\dot{x}=y, \\
\dot{y}=-\frac{1}{10} x\left(x^{2}+2\right)-y x .
\end{array}\right.
$$

Because the system is symmetric with respect to the $x$-axis, i.e. it is invariant under the transformation $(x, t) \rightarrow(-x,-t)$, the linear center at the origin is a center. Moreover, it is the only singularity of the system inside the PoincaréLyapunov disc of degree $(1,2)$. Looking at the behaviour at infinity, Section 2, Figure 2, one sees that there is only one possibility for the global flow of the above system. The system has an unbounded hyperbolic 2-saddle cycle. From Theorem 9 , we indeed know that $m$ has to be at least 3 and $n$ at least 1 for such an unbounded 2-saddle cycle to occur.

\section{Boundaries of period annuli in Liénard systems}

In this section, we describe all possible exterior and interior boundaries of as well bounded as unbounded period annuli. This permits to describe the boundary of all possible Hopf centers, the ones that extend to infinity as well as the ones that do not extend to infinity. Further, we find some sufficient conditions for a Hopf center to be bounded or to be unbounded.

\subsection{Boundaries of period annuli}

Let $\bar{X} \in L^{(m, n)}(D)$, obtained after an appropriate Poincaré-Lyapunov compactification from a Liénard system $X$ of type $(m, n)$ :

$$
X:\left\{\begin{array}{l}
\dot{x}=y, \\
\dot{y}=P(x)+y Q(x),
\end{array}\right.
$$


Preprint submitted at Qualitative Theory of Dynamical Systems. The final publication is available at http://link.springer.com/article/10.1007\\%2Fs12346-008-0019-9

where $P$ and $Q$ are polynomials of respective precise degrees $m$ and $n$. We will describe the possible boundaries of period annuli occuring in $\bar{X}$. We start with some definitions.

An open connected subset $\mathcal{A}$ of the plane filled by closed orbits of $\bar{X}$ is called a period annulus of $X$; we will shortly call it an annulus. We say that the annulus is bounded when $\mathcal{A}$ fits in some compact $K \subset D \backslash \partial D$, if not, $\mathcal{A}$ is said to be unbounded.

Let $\mathcal{A}$ be an annulus of $\bar{X}$. We can provide $\mathcal{A}$ with an order relation $\preceq$. Herefore let $p_{1}, p_{2} \in \mathcal{A}$. Then, one can find two closed orbits of $\bar{X}, \gamma_{1} \subset \mathcal{A}$ and $\gamma_{2} \subset \mathcal{A}$ such that $p_{1} \in \gamma_{1}$ and $p_{2} \in \gamma_{2}$. Denote $A_{1}$ and $A_{2}$ as the regions enclosed by $\gamma_{1}$ and $\gamma_{2}$ respectively. We say that $p_{1} \preceq p_{2}$ if and only if $A_{1} \subseteq A_{2}$. If both $p_{1} \preceq p_{2}$ and $p_{2} \preceq p_{1}$, then we say that $p_{1}$ is equivalent to $p_{2}$, denoted as $p_{1} \sim p_{2}$; this happens when $p_{1}$ and $p_{2}$ both belong to the same closed orbit.

We say that a sequence $\left(p_{n}\right)_{n \in \mathbb{N}}$ in $\mathcal{A}$ is monotonically increasing in $\mathcal{A}$ if $p_{i} \preceq$ $p_{i+1}, \forall i \in \mathbb{N}$. If $p_{i+1} \preceq p_{i}, \forall i \in \mathbb{N}$, we say that the sequence is monotonically decreasing in $\mathcal{A}$.

We define the exterior boundary of $\mathcal{A}$ as

$$
\partial^{e} \mathcal{A}=\left\{q \in \partial \mathcal{A} \mid \exists\left(p_{n}\right)_{n} \text { monotonically increasing in } \mathcal{A}: p_{n} \rightarrow q\right\},
$$

and the interior boundary of $\mathcal{A}$ as

$$
\partial^{i} \mathcal{A}=\left\{q \in \partial \mathcal{A} \mid \exists\left(p_{n}\right)_{n} \text { monotonically decreasing in } \mathcal{A}: p_{n} \rightarrow q\right\} .
$$

Clearly $\partial^{e} \mathcal{A} \cap \partial^{i} \mathcal{A}=\emptyset$. Any periodic orbit of $\bar{X}$ intersects the $x$-axis in exactly two points. In particular the intersection of $\mathcal{A}$ with the $x$-axis is the union of two open intervals $] a, b[\cup] c, d[$ lying on the $x$-axis, see Figure 16 . Notice, when $a$ or $d$ lie on the circle at infinity, the annulus $\mathcal{A}$ is unbounded.

For each $x \in] a, d\left[\right.$, the sections $\Sigma_{x}^{+}=\{(x, y) \in D \mid y>0\}$ and $\Sigma_{x}^{-}=$ $\{(x, y) \in D \mid y<0\}$ both intersect $\mathcal{A}$ in an unique open interval:

$$
\begin{aligned}
& \left.\mathcal{A} \cap \Sigma_{x}^{+}=\{x\} \times\right] p_{x}^{+}, q_{x}^{+}[, \\
& \left.\mathcal{A} \cap \Sigma_{x}^{-}=\{x\} \times\right] q_{x}^{-}, p_{x}^{-}[,
\end{aligned}
$$

where for each $x, p_{x}^{ \pm}$and $q_{x}^{ \pm}$are uniquely determined, with $\left(x, p_{x}^{ \pm}\right)$and $\left(x, q_{x}^{ \pm}\right)$ lying either in the interior of the disc $D$ or on the circle at infinity.

Proposition 10 Suppose $\mathcal{A}$ is a period annulus of $\bar{X} \in L^{(m, n)}(D)$ that intersects the $x$-axis in $] a, b[\cup] c, d[$. Consider

$$
\begin{array}{ll}
\Gamma_{e}^{+}=\left\{\left(x, q_{x}^{+}\right) \mid x \in\right] a, d[\}, & \Gamma_{i}^{+}=\left\{\left(x, p_{x}^{+}\right) \mid x \in\right] b, c[\}, \\
\Gamma_{e}^{-}=\left\{\left(x, q_{x}^{-}\right) \mid x \in\right] a, d[\}, & \Gamma_{i}^{-}=\left\{\left(x, p_{x}^{-}\right) \mid x \in\right] b, c[\},
\end{array}
$$

with $p_{x}^{ \pm}, q_{x}^{ \pm}$defined as in (12). Then the exterior and interior boundaries of $\mathcal{A}$ are invariant subsets for the flow of $\bar{X}$, given by

$$
\begin{aligned}
& \partial^{e} \mathcal{A}=\{(a, 0)\} \cup\{(d, 0)\} \cup \Gamma_{e}^{+} \cup \Gamma_{e}^{-}, \\
& \partial^{i} \mathcal{A}=\{(b, 0)\} \cup\{(c, 0)\} \cup \Gamma_{i}^{+} \cup \Gamma_{i}^{-} .
\end{aligned}
$$

Further $\{(a, 0)\} \cup\{(d, 0)\} \cup \Gamma_{e}^{ \pm}$and $\{(b, 0)\} \cup\{(c, 0)\} \cup \Gamma_{i}^{ \pm}$are all continuous graphs. 


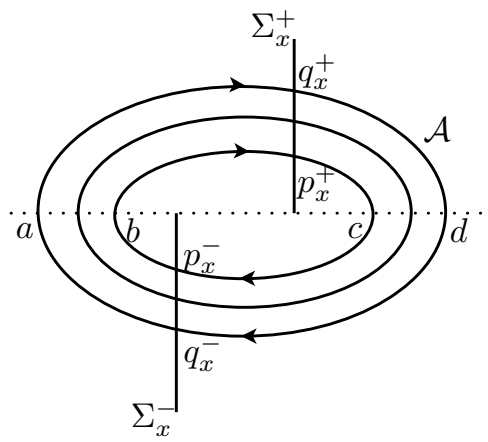

Figure 16: An annulus $\mathcal{A}$ of $\bar{X}$, the dotted line represents the $x$-axis.

Proof: Let us first prove the invariance, for the flow $\phi_{t}: D \mapsto \mathbb{R}^{2}$ of $\bar{X}$, of the exterior and interior boundary of $\mathcal{A}$. Let $p \in \partial^{e} \mathcal{A}\left(\right.$ resp. $p \in \partial^{i} \mathcal{A}$ ), then there exists a sequence $\left(p_{n}\right)_{n}$ in $\mathcal{A}$ that is monotonically increasing (resp. decreasing) in $\mathcal{A}$ and that converges to $p$. Because of the continuity of the flow $\phi_{t}$ of $\bar{X}$, one immediately concludes that $\left(\phi_{t}\left(p_{n}\right)\right)_{n}$ is a monotonically increasing (resp. decreasing) sequence in $\mathcal{A}$ converging to $\phi_{t}(p)$. We proceed by proving the equalities in (14). Clearly the boundary of $\mathcal{A}$ is given by:

$$
\mathcal{F}:=\{(a, 0),(b, 0),(c, 0),(d, 0)\} \cup \Gamma_{e}^{ \pm} \cup \Gamma_{i}^{ \pm} .
$$

Indeed, one easily verifies that $\forall p \in \mathcal{F}, \forall \varepsilon>0: B(p, \varepsilon) \cap \mathcal{A} \neq \emptyset$ and $B(p, \varepsilon) \cap$ $\mathcal{A}^{c} \neq \emptyset$. Since $\partial^{e} \mathcal{A} \cap \partial^{i} \mathcal{A}=\emptyset$, it clearly suffices to prove that

$$
\{(a, 0)\} \cup\{(d, 0)\} \cup \Gamma_{e}^{+} \cup \Gamma_{e}^{-} \subset \partial^{e} \mathcal{A}
$$

and

$$
\{(b, 0)\} \cup\{(c, 0)\} \cup \Gamma_{i}^{+} \cup \Gamma_{i}^{-} \subset \partial^{i} \mathcal{A},
$$

for showing (14). We prove the inclusion concerning the exterior boundary, the other one can be proved completely analogously.

Suppose $\left(x, q_{x}^{+}\right) \in \Gamma_{e}^{+}$, then the sequence $\left(x, q_{x}^{+}-\frac{1}{n}\right)_{n \geq N}$, with $N$ big enough, lies in $\mathcal{A}$ and converges to $\left(x, q_{x}^{+}\right)$. Moreover, one easily sees that $\left(x, q_{x}^{+}-\frac{1}{n}\right)_{n}$ is a monotonically increasing sequence in $\mathcal{A}$. It follows that $\Gamma_{e}^{+} \subset \partial^{e} \mathcal{A}$. Similar $\Gamma_{e}^{-} \subset \partial^{e} \mathcal{A}$. One also sees that $(a, 0)$ and $(d, 0)$ belong to $\partial^{e} \mathcal{A}$ by choosing appropriate sequences in $\mathcal{A}$ that are lying on the $x$-axis and that converge to $(a, 0)$ and $(d, 0)$.

Concerning the statement of the continuity, we will only treat the interior boundary. The exterior boundary can be treated in the same way. In fact in the unbounded case, the result follows from the classification that we will give in Theorem 11 and which does not rely on the previous knowledge of the continuity in the unbounded case.

Let $\mathcal{G}=\{(a, 0)\} \cup\{(d, 0)\} \cup \Gamma_{i}^{+}$. Then $\mathcal{G}$ is the graph of the function

$$
P^{+}: x \mapsto p_{x}^{+}
$$

where we set $p_{x}^{+}=0$ when $x=a$ or $x=d$. We prove that $P^{+}$is continuous in any $x_{0} \in[a, d]$. Suppose first $\left(x_{0}, P^{+}\left(x_{0}\right)\right)$ is a regular point of the flow of 
Preprint submitted at Qualitative Theory of Dynamical Systems. The final publication is available at http://link.springer.com/article/10.1007\\%2Fs12346-008-0019-9

$\bar{X}$. Because the interior boundary is invariant for the flow of $\bar{X}$, it is clear that for $x \in[a, d]$ and near $x_{0}$, the graph of $P^{+}$is given by a connected piece of the regular orbit of $X$ passing through $\left(x_{0}, P^{+}\left(x_{0}\right)\right)$ such that $P^{+}$is continuous at $x_{0}$. If $\left(x_{0}, P^{+}\left(x_{0}\right)\right)$ is a singularity, then it has to contain a hyperbolic sector induced by the annulus $\mathcal{A}$. From the list of singularities in Section 2, it is clear that the boundary of such a hyperbolic sector is always continuous. Analogously, one shows that $\{(a, 0)\} \cup\{(d, 0)\} \cup \Gamma_{i}^{-}$is a continuous graph.

We can now completely describe all possible boundaries of annuli occuring in a Liénard system $\bar{X} \in L^{(m, n)}(D)$. Let us first treat the exterior boundaries.

Theorem 11 Suppose $\mathcal{A}$ is a period annulus of $\bar{X} \in L^{(m, n)}(D)$, then

i. if $\mathcal{A}$ is bounded, then $\partial^{e} \mathcal{A}$ is a loop, as in Figure 17 (a) or (b), or a cycle with 2 singularities, as in Figure 17 (c). The singularities on the exterior boundary can only be saddles (as well hyperbolic as non-hyperbolic), saddle-nodes (as well semi-hyperbolic as nilpotent) or cusps as described in Section 2,

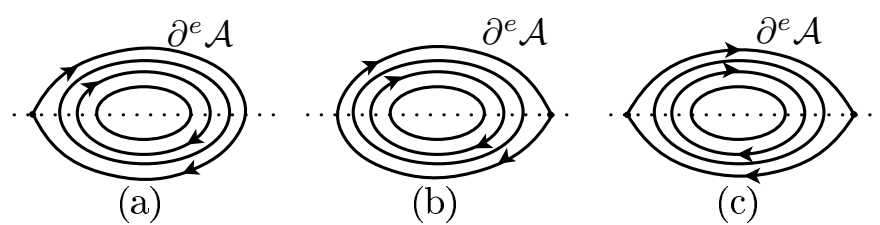

Figure 17: Possible exterior boundaries of a bounded annulus $\mathcal{A}$ of $\bar{X}$.

ii. if $\mathcal{A}$ is not bounded, then one of the below mentioned conditions have to be satisfied (as in Section 2, A denotes the highest order coefficient of the polynomial $P$ in (2)):

(a) When

$$
m=2 n+1, A>\frac{1}{4(n+1)} \text { or } m>2 n+1, m \text { odd and } A=1,
$$

then $\partial^{e} \mathcal{A}$ is given by a periodic orbit lying at infinity, see Figure 18 (a).

(b) When

$$
m<2 n+1, m \text { and } n \text { odd, and } A=1,
$$

the exterior boundary looks like in Figure 18 (b) containing at infinity two semi-hyperbolic saddles $s_{1}$ and $s_{2}$ such that $s_{1}$ (resp. $s_{2}$ ) has an unstable (resp. stable) separatrix lying at infinity and an attractive (resp. repelling) center separatrix in $D \backslash \partial D$.

(c) When

$$
m=2 n+1, n \text { odd }, 0<A \leq \frac{1}{4(n+1)},
$$

$\partial^{e} \mathcal{A}$, looks like in Figure $18(c)$. When $A=1 /(4(n+1))$, the singularities at infinity are saddle-nodes of which the center separatrix lies 
Preprint submitted at Qualitative Theory of Dynamical Systems. The final publication is available at http://link.springer.com/article/10.1007\\%2Fs12346-008-0019-9

at infinity. The behaviour on the hyperbolic seperatric is attractive for $s_{1}$, while it is repelling for $s_{2}$. When $A<1 /(4(n+1)), s_{1}$ and $s_{2}$ are both hyperbolic saddles.

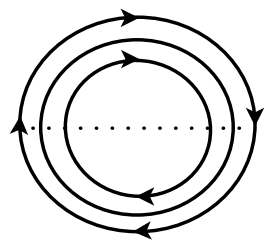

(a)

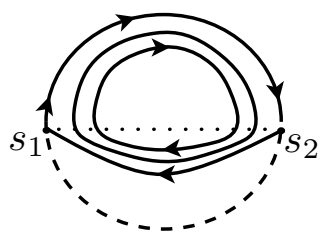

(b)

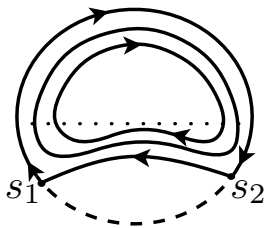

(c)

Figure 18: Possible exterior boundaries of an unbounded annulus $\mathcal{A}$ of $\bar{X}$.

Proof: From Proposition 10, one concludes that the exterior boundary of $\mathcal{A}$ is an invariant set for the flow of $\bar{X}$, being (at least in the bounded case) the union of two continuous graphs, one lying in the half plane $\{y \geq 0\}$ and one lying in $\{y \leq 0\}$. Moreover the graphs are given by

$$
\{(a, 0)\} \cup\{(d, 0)\} \cup \Gamma_{e}^{+} \quad \text { and } \quad\{(a, 0)\} \cup\{(d, 0)\} \cup \Gamma_{e}^{-},
$$

with $\Gamma_{e}^{+} \subset\{y>0\}, \Gamma_{e}^{-} \subset\{y<0\}$, defined in (13), lying enterily in the region $a<x<d$.

In particular if $\mathcal{A}$ is bounded, then $\mathcal{A}$ lies enterily in some compact $K \subset$ $D \backslash \partial D$ such that the only possible singularities of $\bar{X}$ lying on $\partial^{e} \mathcal{A}$ are $(a, 0)$ and $(d, 0)$. This completely determines the structure of $\partial^{e} \mathcal{A}$. Furthermore, because of the analyticity of the Liénard system $X, \partial^{e} \mathcal{A}$, has to contain at least one singularity.

If only $(a, 0)$ (resp. $(d, 0))$ is a singularity, then $\partial^{e} \mathcal{A}$ is a loop consisting of the singulariy $(a, 0)$ (resp. $(d, 0))$ and a regular orbit of $\bar{X}$ given by $\Gamma_{e}^{+} \cup \Gamma_{e}^{-} \cup\{(d, 0)\}$ (resp. $\Gamma_{e}^{+} \cup \Gamma_{e}^{-} \cup\{(a, 0)\}$. If both $(a, 0)$ and $(d, 0)$ are singularities, the exterior boundary of $\mathcal{A}$ consists of the two singularities $(a, 0)$ and $(d, 0)$ together with regular orbits $\Gamma_{e}^{+}$and $\Gamma_{e}^{-}$connecting them.

Obvious the annulus $\mathcal{A}$ induces a hyperbolic sector on the singularities of $\bar{X}$ lying on its exterior boundary. So referring to Section 2, the only possibilities for the singularities are saddles, saddle-nodes and cusps.

Suppose now $\mathcal{A}$ is unbounded. Then $\partial^{e} \mathcal{A}$ includes the whole circle at infinity or part of it. When it includes the whole circle at infinity, the boundary is given by a regular periodic orbit at infinity. When it includes partly the circle at infinity, $\partial^{e} \mathcal{A}$ has to contain two singularities, $s_{1}$ and $s_{2}$, at infinity, with a hyperbolic sector induced by the annulus $\mathcal{A}$. Referring to the study at infinity of $\bar{X} \in L^{(m, n)}(D)$ in Section 2, it is clear that only the graphics in Figure 18 can occur as exterior boundaries of unbounded annuli.

In case (a), $\partial^{e} \mathcal{A}$ contains no singularities and is given by a periodic orbit at infinity. In case (b), $(a, 0)$ and $(d, 0)$ are given by singularities of $\bar{X}$ at infinity, $\Gamma_{e}^{+}$is a regular orbit lying at infinity and $\Gamma_{e}^{-}$is a regular orbit that lies completely under the $x$-axis. In case (c), the singularities are lying on $\Gamma_{e}^{-},(a, 0)$ and $(d, 0)$ are part of a regular orbit lying at infinity between the singularities $s_{1}$ and $s_{2}$. 
Preprint submitted at Qualitative Theory of Dynamical Systems. The final publication is available at http://link.springer.com/article/10.1007\\%2Fs12346-008-0019-9

Concerning the interior boundaries, we have the following theorem.

Theorem 12 The interior boundary of some period annulus $\mathcal{A}$ of $\bar{X} \in L^{(m, n)}(D)$, is given by

i. a center, or

ii. the union of the two bounded (i.e. not intersecting $\partial D$ ) continuous graphs

$$
\{(a, 0)\} \cup\{(d, 0)\} \cup \Gamma_{i}^{+} \quad \text { and } \quad\{(a, 0)\} \cup\{(d, 0)\} \cup \Gamma_{i}^{-} \text {, }
$$

lying respectively in the half planes $\{y \geq 0\}$ and $\{y \leq 0\}$ and containing at least one singularity of $\bar{X}$. Concerning the type of the singularities, we have the following:

(a) the zeros of the continuous graph $\Gamma_{i}^{+}$(resp. $\Gamma_{i}^{-}$) are singularities that can only be saddles or one of the singularities presented in Section 3, Figure 9 and Figure 10 (resp. Figure 11),

(b) the intersections between $\Gamma_{i}^{+}$and $\Gamma_{i}^{-}$are saddles,

(c) if $(a, 0)$ (resp. $(d, 0))$ is a singularity, then it has to be a cusp of up-up (resp. down-down) type.

Proof: From Proposition 10, one concludes that the interior boundary of $\mathcal{A}$ is an invariant set for the flow of $\bar{X}$, being the union of two continuous graphs, one lying in the half plane $\{y \geq 0\}$ and one lying in $\{y \leq 0\}$. The graphs are given by

$$
\{(b, 0)\} \cup\{(c, 0)\} \cup \Gamma_{i}^{+} \text {and }\{(b, 0)\} \cup\{(c, 0)\} \cup \Gamma_{i}^{-},
$$

with $\Gamma_{i}^{+} \subset\{y \geq 0\}, \Gamma_{i}^{-} \subset\{y \leq 0\}$, defined in (13), lying enterily in the region $b<x<c$. The two graphs intersect the $x$-axis in $(b, 0)$ and $(c, 0)$ and possibly other points, with abscis lying in $] b, c[$.

Whether the annulus is bounded or unbounded, $\partial^{i} \mathcal{A}$ is clearly situated in $D \backslash \partial D$. Because of the analyticity of the Liénard system $X, \partial^{i} \mathcal{A}$, has to contain at least one singularity.

If $b=c$, then obviously $\partial^{i} \mathcal{A}$ has to be a center. If $b \neq c$, then $\partial^{i} \mathcal{A}$ is a graphic being the union of the two continuous graphs in (15). If $(b, 0)$ (resp. $(c, 0))$ is a singularity it clearly has to contain an upgoing (resp. downgoing passage) and a hyperbolic sector lying left (resp. right) of this vertical passage. Referring to the study of the singularities in Section 2, one sees that this is only possible when $(b, 0)$ (resp. $(c, 0)$ ) is a cusp of up-up type (resp. down-down type). The singularities lying on $\Gamma_{i}^{+}\left(\operatorname{resp} . \Gamma_{i}^{-}\right)$, and with abscis in $] b, c[$, clearly contain a lr-passage (resp. rl-passage) determining the type of the singularities (see Section 3, Figures 9, 10 and 11).

From Theorem 11, it is clear that the exterior boundaries of bounded (resp. unbounded) annuli form a subset of the list of bounded (resp. unbounded) limit periodic sets that are inner polycycles. An inner polycycle is a cycle for which a return map can be defined in the open region enclosed by the cycle. Similarly, from Theorem 12, the interior boundaries of the annuli form a subset of the list of bounded limit periodic set that are outer polycycles. An outer polycycle is 
Preprint submitted at Qualitative Theory of Dynamical Systems. The final publication is available at http://link.springer.com/article/10.1007\\%2Fs12346-008-0019-9

a cycle for which the return map can be defined in the exterior of the region enclosed by the cycle.

Not every limit periodic set is the boundary of some annulus. There are some restrictions on the structure of a limit periodic set making it the boundary of some annulus. The question remains whether every boundary of some annulus is indeed a limit periodic set. Herefore, one has to search an appropriate unfolding in $L^{(m, n)}(D)$, of a Liénard system with an annulus $\mathcal{A}$, producing a limit cycle that can be put arbitrarily close to the exterior (resp. interior) boundary of $\mathcal{A}$ for the Hausdorff metric. At the moment, we won't focus our attention to this question. Let us instead, as a consequence of the former results, give a complete description of the boundary of any Hopf center occuring in a Liénard system.

\subsection{Boundaries of Hopf centers}

We say that $\bar{X}$ has a Hopf center at $s$ if $s$ is a linear center of $\bar{X}$ of which an open neighbourhood is filled by closed orbits of $\bar{X}$. In particular $\bar{X}$ contains an annulus $\mathcal{A}$ with $\partial^{i} \mathcal{A}=\{s\}$. We say that $s$ is a bounded Hopf center if $\mathcal{A}$ is bounded. When $\mathcal{A}$ is unbounded, $s$ is an unbounded Hopf center. Evidently a Hopf center $s$ can never lie at infinity.

In this section we will describe all possible boundaries of, as well bounded as unbounded, Hopf centers. We find some sufficient conditions for a Hopf center to be bounded or unbounded.

From Theorem 11, we can describe all possible exterior boundaries of the annulus $\mathcal{A}$ of $\bar{X} \in L^{(m, n)}(D)$ occuring when $\bar{X}$ has a Hopf center $s$. We come to three possibilities, shown in Figure 19. The singularities on $\partial^{e} \mathcal{A}$ can only be saddles, saddle-nodes or cusps.

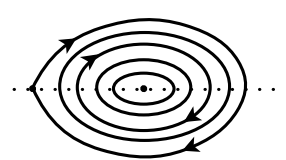

(a)

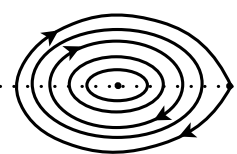

(b)

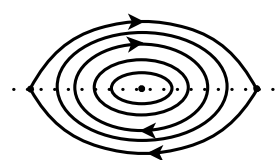

(c)

Figure 19: Possible bounded Hopf centers of $\bar{X}$. The Hopf centers are bounded by a loop in (a) and (b) and a cycle with two singularities in (c).

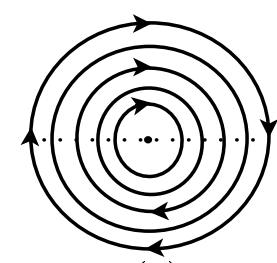

(a)

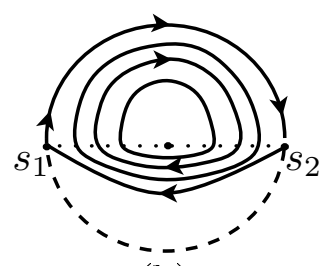

(b)

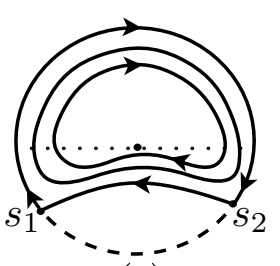

(c)

Figure 20: Possible unbounded Hopf centers of $\bar{X}$. 
Preprint submitted at Qualitative Theory of Dynamical Systems. The final publication is available at http://link.springer.com/article/10.1007\\%2Fs12346-008-0019-9

For an unbounded Hopf center, we have the options illustrated in Figure 20. As Theorem 11 states, case (a) can only occur when $m=2 n+1, A>$ $1 /(4(n+1))$; or when $m>2 n+1, m$ odd, $A=1$. Case (b) can only occur when $m<2 n+1, m$ and $n$ odd, $A=1$. The singularities $s_{1}$ and $s_{2}$ at infinity are semihyperbolic saddles where $s_{1}$ (resp. $s_{2}$ ) has an unstable (resp. stable) separatrix lying at infinity and an attractive (resp. repelling) center separatrix in $D \backslash \partial D$. Case (c) can only occur when $m=2 n+1, n$ odd and $0<A \leq 1 /(4(n+1))$. When $A=1 /(4(n+1))$ the singularities at infinity are given by saddle-nodes of which the center separatrix lies at infinity. The behaviour on the hyperbolic separatrix is attractive for $s_{1}$, while it is repelling for $s_{2}$. When $A<1 /(4(n+1))$, the singularities at infinity are hyperbolic saddles.

Let us now consider the question whether a Hopf center is bounded or unbounded. Using the above classification of unbounded Hopf centers, one easily proves the following theorem.

Theorem 13 Suppose $\bar{X} \in L^{(m, n)}(D)$ has a Hopf center $s$ in $D \backslash \partial D$, then it is unbounded if and only if $X$ has an unique singularity. Such an unbounded Hopf center can only occur in the cases:

i. $m=2 n+1, A>1 /(4(n+1))$,

ii. $m=2 n+1, n$ odd, $0<A \leq 1 /(4(n+1))$,

iii. $m>2 n+1, m$ odd, $A=1$,

iv. $m<2 n+1, m$ odd, $n$ odd, $A=1$.

For verifying whether a given singularity $s$ of $\bar{X} \in L^{(m, n)}(D)$ is a Hopf center, one can use the following theorem. Notice that after a suitable translation, $s$ can supposed to lie at the origin.

Theorem 14 (C. Christopher [1]) Let $\bar{X} \in L^{(m, n)}(D)$ obtained after an appropriate Poincaré-Lyapunov compactification from a Liénard system $X$ (11), with $P^{\prime}(0)<0$, then $\bar{X}$ has a center at the origin if and only if there exists a polynomial, $M(x)=x^{2}+O\left(x^{3}\right)$ and polynomials $k(z), l(z)$ with $k(0)=l(0)=0$ such that $X$ is given by:

$$
\left\{\begin{array}{l}
\dot{x}=y-k(M(x)), \\
\dot{y}=-\frac{d}{d x}(l(M(x))) .
\end{array}\right.
$$

The above theorem completely characterises the Hopf centers in Liénard systems. Another tool for characterising Hopf centers are Lyapunov coefficients. For a general introduction to the Lyapunov coefficients as well as an algorithm to compute them, we refer to [4]. In particular $X$ reads like (16) if and only if all Lyapunov coefficient at $s$ are zero.

Theorem 15 Suppose $\bar{X} \in L^{(m, n)}(D)$, obtained after an appropriate PoincaréLyapunov compactification from a Liénard system $X$ that is given by:

$$
\left\{\begin{aligned}
\dot{x} & =y-k(M(x)), \\
\dot{y} & =-\frac{d}{d x}(l(M(x))),
\end{aligned}\right.
$$

for some polynomials $M(x), k(z), l(z)$ with $M(x)=x^{2}+O\left(x^{3}\right), k(0)=0, l(0)=$ $0, l^{\prime}(0)>0$ and the degree of $M$ odd. Then the origin is a bounded Hopf center. 
Preprint submitted at Qualitative Theory of Dynamical Systems. The final publication is available at http://link.springer.com/article/10.1007\\%2Fs12346-008-0019-9

Proof: From Theorem 14, we already know that the origin is a Hopf center. Using the previous theorem, we only have to show that the origin is not the only singularity of $\bar{X}$ in $D \backslash \partial D$.

The degree of $M$ is odd, thus

$$
M(x)=x^{2}+m_{1} x^{3}+\cdots+m_{2 k-1} x^{2 k+1},
$$

for some $k \geq 1$. Therefore:

$$
M^{\prime}(x)=x\left(2+3 m_{1} x+\cdots(2 k+1) m_{2 k-1} x^{2 k-1}\right),
$$

implying that $M^{\prime}(x)=x M_{0}(x)$ with $M_{0}(0) \neq 0$ and the degree of $M_{0}$ odd. So $M_{0}$ has to possess a zero $x_{0} \neq 0$ implying that $X$ has a singularity not lying at the origin.

\section{References}

[1] C. Christopher, An algebraic approach to the classificiation of centers in polynomial Liénard systems, Journal of Mathematical Analysis and Applications 229 (1999), no. 1, 319-329.

[2] F. Dumortier, Compactification and desingularisation of spaces of polynomial Liénard equations, Journal of Differential Equations 224 (2006), no. 2, 296-313.

[3] F. Dumortier and C. Herssens, Polynomial Liénard equations near infinity, Journal of Differential Equations 153 (1999), no. 1, 1-29.

[4] F. Dumortier, J. Llibre, and J.C.Artes, Qualitative theory of planar differential systems, Springer-Verlag, 2006.

[5] J.-P. Françoise and C.C. Pugh, Keeping track of limit cycles, Journal of Differential Equations 65 (1986), no. 1, 139-157.

[6] L. Perko, Differential equations and dynamical systems, Springer-Verlag, 1986.

[7] R. Roussarie, A note on finite cyclicity property and hilbert's 16th problem, Lecture Notes in Mathematics 1331 (1988), 161-168.

[8] R. Roussarie, Bifurcations of planar vector fields and Hilbert's sixteenth problem, Birkhauser-Verlag, 1998. 